\title{
Götz Rohwer
}

»Kritik der ökonomischen Vermunft《 Anmerkungen zu einem Buch von André Gorz

Zusammenfassung: Der Aufsatz beschäftigt sich mit André Gorz' neuem Buch "Kritik der ökonomischen Vernunft. Sinnfragen am Ende der Arbeitsgesellschaft . Gorz' These eines langfristigen Rückgangs in der Nachfrage nach Arbeit wird kritisiert. Weiterhin erfolgt eine Auseinandersetzung mit Gorz' Überlegungen, daß es Grenzen »ökonomischer Rationalisierung «, d.h. der Verwandlung menschlichen Lebens in kapitalistisch organisierte Lohnarbeit und Konsum kapitalistisch produzierter Güter und Dienstleistungen, gibt. Es wird dargelegt, daß es wichtig ist, analytische und normative Behauptungen über Grenzen »ökonomischer Rationalisierung « zu unterscheiden.

\section{Einleitung}

»Mit einem Wort: es geht um den Übergang von einer produktivistischen oder Arbeitsgesellschaft zu einer Gesellschaft der befreiten Zeit, in der Kultur und Gesellschaftlichkeit das Ökonomische überwiegen. «In dieser neuen Gesellschaft »wird die fortschreitende Verringerung der ökonomisch zweckbestimmten Arbeit ein Übergewicht der autonomen Tätigkeiten möglich gemacht haben.« - In seinem neuen Buch »Kritik der ökonomischen Vernunft. Sinnfragen am Ende der Arbeitsgesellschaft « ${ }^{1}$ hat sich André Gorz vorgenommen, diese Auffassung erneut darzulegen und ihr eine umfassende Begründung zu geben. Das Buch enthält zunächst eine Kritik an der marxistischen Theorietradition und der in ihr begründeten Sozialismuskonzeption. Mit dieser Kritik versucht Gorz zu zeigen, daß die in dieser Tradition entwickelte Vorstellung einer umfassenden (ökonomischen) Rationalisierung der gesellschaftlichen Lebensverhältnisse aufgegeben werden sollte zugunsten eines neuen Ideals einer $»$ Befreiung von der Arbeit «, d.h. von ihrer ökonomischen Zweckbestimmtheit. Zur weiteren Begründung dieser Auffassung bemüht sich Gorz dann vor allem darum, Grenzen ökonomischer Rationalität aufzuzeigen. Die hierfür zentrale Argumentationsstrategie des Buches besteht darin, Krisenphänomene, wie sie in der gegenwärtigen ökonomischen und gesellschaftlichen Entwicklung sichtbar werden, als Ausdruck bzw. Folge von Grenzen der Möglichkeit einer fortgesetzten ökonomischen Rationalisierung der gesellschaftlichen Lebensverhältnisse zu interpretieren. Diese Interpretation begründet schließlich Gorz' Vorschlag einer langfristig konzipierten Arbeitszeitverkürzung, verbunden mit Maßnahmen zur Umverteilung von Arbeit und durch Arbeitsverpflichtungen zu sichernden Einkommensgarantien.

Die Argumentationsstrategie beruht wesentlich auf einem Begriff »ökonomischer 
Rationalität«. Vielleicht deshalb wird dieser Begriff von Gorz nirgendwo zusammenhängend definiert. Gemeint ist damit ungefähr folgendes: daß Lebenstätigkeiten der Menschen einem ökonomischen, d.h. einem monetär vermittelten Kosten-NutzenKalkiil unterworfen werden mit dem Ziel, ein möglichst optimales Kosten-NutzenVerhältnis zu erreichen. Die Entwicklung des Kapitalismus ist für Gorz, wobei er gelegentlich explizit an Max Weber anknüpft, im wesentlichen gleichbedeutend mit einem Prozeß der Durchsetzung ökonomischer Rationalität, kurz gesagt: mit ökonomischer Rationalisierung. $\gg$ Der Kapitalismus wurde zum Ausdruck der endlich von allen Fesseln befreiten ökonomischen Rationalität. «(S. 176) Damit ist vor allem gemeint, daß die Entwicklung einer kapitalistischen Ökonomie als ein fortgesetzter Prozeß der Verwandlung menschlichen Lebens in kapitalistisch organisierte Lohnarbeit und Konsum kapitalistisch produzierter Güter und Dienstleistungen betrachtet werden soll. Mit dem Begriff »ökonomische Rationalisierung « soll auf diesen Prozeß verwiesen werden. Diese Betrachtungsweise führt nun sogleich zu einer der Hauptfragen des Buches: Gibt es Grenzen ökonomischer Rationalisierung? Oder in etwas anderer Formulierung: Gibt es Grenzen einer kapitalistischen Ökonomie? Gorz will zeigen, daß es solche Grenzen gibt, »das es ontologische, existentielle Grenzen der Rationalisierung gibt und daß diese Grenzen nur von vernunftwidrigen PseudoRationalisierungen übertreten werden können, in denen sich die Rationalisierung in ihr Gegenteil verkehrt. In der Eingrenzung der Sphäre dessen, was rationalisierbar ist, liegt eines der Hauptziele dieses Buches.« (S. 14)

Ich will mich im folgenden vor allem mit dieser These auseinandersetzen. Denn einerseits ist ihre Bedeutung offensichtlich, andererseits jedoch auch ihre Problematik. Gorz' Argumentationsstrategie beruht darauf, sinnvolle und vernünftige von nicht sinnvollen und nicht-vernünftigen Möglichkeiten ökonomischer Rationalisierung zu unterscheiden. Aber wer entscheidet über diese Unterscheidung? Gorz macht Vorschläge für eine solche Unterscheidung und begründet sie; insofern ist es interessant und wichtig, sich mit ihnen auseinanderzusetzen. Aber dabei bleibt weitgehend offen, wie in Wirklichkeit die Entscheidungsprozesse verfaßt sind, die die Expansion einer kapitalistischen Ökonomie vermitteln und dadurch zugleich fortgesetzt neue Möglichkeiten ökonomisçher Rationalisierung demonstrieren. Das Problem besteht also, etwas anders formuliert, darin, daß Gorz im wesentlichen nur normativ - kulturkritisch moralisierend - argumentiert, daß mit einer solchen Argumentation aber über effektive Grenzen einer kapitalistischen Ökonomie nichts ausgesagt werden kann. Wobei ich betonen möchte, daß es sich hierbei um ein sehr grundlegendes Problem der Kapitalismuskritik handelt; nicht nur deshalb, weil es schwer ist, sich über eine normative Kritik zu verständigen, sondern vor allem weil eine kapitalistische Ökonomie gewissermaßen über die Fähigkeit verfügt, solche Kritik gegenstandslos zu machen, indem sie die Bedingungen ihrer Formulierbarkeit - moralisch und kulturell geprägte Milieus und Biographien - zerstört bzw. überlebt. Gerade weil ich mit Gorz' Bemühen, Grenzen ökonomischer Rationalisierung zu finden, weitgehend übereinstimme, finde ich es wichtig, ihn mit diesem Einwand - jedenfalls einer naheliegenden Vermutung - zu konfrontieren: daß seine Kapitalismuskritik (und infolgedessen 
auch seine Sozialismuskonzeption) unzureichend ist, weil sie gewissermaßen die soziale Maclıt - und ihre institutionelle Verfaßtheit - verfehlt, die eine kapitalistische Ökonomie in die Lage versetzt, Ideologie- und Kulturkritik obsolet zu machen.

\section{Kapitalistische Ökonomie und notwendige Arbeit}

Gorz versucht, Grenzen ökonomischer Rationalisierung vor allem in zweierlei Hinsicht aufzuzeigen. Erstens dadurch, daß er ökonomische Rationalisierung als einen Prozeß interpretiert, bei dem durch Einsatz technischen Fortschritts langfristig immer weniger Arbeit notwendig wird. Zweitens, indem er sich zu zeigen bemuiht, daß ökonomische Rationalisierung an Sinnvoraussetzungen gebunden ist, so daß zahlreiche Lebenstätigkeiten einem Zugriff ökonomischer Rationalität nicht unterworfen werden können. In diesem Abschnitt will ich mich mit dem ersten dieser beiden Gedankengänge beschäftigen. Er liefert zugleich einen Zugang zu Gorz' Interpretation der gegenwärtigen gesellschaftlichen Entwicklung als den Beginn eines Endes der bisherigen »Arbeitsgesellschaft«, d.h. einer Gesellschaft, in der die Erwerbstätigkeit und das ihren Sinn vermittelnde Erwerbsstreben das gesellschaftliche Leben beherrschen. Die wesentliche Behauptung, die diese Interpretation begründen soll, lautet, da $B$ die gegenwärtige Arbeitslosigkeit der erste sichtbare Ausdruck einer langfristig immer mehr abnehmenden Nachfrage nach Arbeit ist. So heißt es etwa:

Die »Reduktion gesellschaftlich notwendiger Arbeit auf ein Minimum ist heute in vollem Gange: Die Industriegesellschaften produzieren eine wachsende Menge von Reichtümern mit abnehmenden Arbeilsmengen « (S. 134). »In der Industrie ebenso wie im klassischen Dienstleistungssektor schreitet die Schrumpfung des Arbeitskräftebedarfs mit immer größerer Geschwindigkeit voran« (S. 313).

Mit empirischen Hinweisen kann aber natürlich die intendierte These nicht begründet werden; denn es ist durchaus vorstellbar (und bekanntlich auch als ein wirtschaftspolitisches Ziel formulierbar), daß die gegenwärtige Arbeitslosigkeit nur Ausdruck einer vorübergehenden Krise ist, nicht jedoch eine Folge systematisch und dauerhaft wirksamer Wachstumsgrenzen. ${ }^{2}$ Gorz interpretiert dagegen die anhaltende Arbeitslosigkeit als Folge eines langfristigen ökonomischen Rationalisierungsprozesses, in dem durch technischen Fortschritt Arbeit zunehmend weniger notwendig wird. Infolgedessen stellen sich zwei Fragen: Stimmt es, daß Arbeit zunehmend weniger notwendig wird; was ist in diesem Zusammenhang mit »notwendig « gemeint? Und welche Rolle spielt dabei der technische Fortschritt?

Das erste der beiden Probleme wird exemplarisch an folgender Formulierung deutlich: »Was aber zu Anfang des letzten Jahrhunderts eine Utopie war, ist dies heute teilweise bereits nicht mehr: der gesamtgesellschaftliche Produktionsprozeß, die Wirtschaft benötigen immer weniger Lohnarbeit. Die Unterordnung aller anderen menschlichen Tätigkeiten und Ziele unter die Lohnarbeit und die ökonomischen Zwecke verliert damit ihre Notwendigkeit und ihren Sinn. «(S. 312 f.) Denn man muß sich doch sogleich fragen, wofür Lohnarbeit überhaupt »notwendig « ist; und diese Frage läßt sich offenbar aus ganz unterschiedlichen Perspektiven stellen und beant- 
worten. Zunächst aus der Sicht der Erwerbstätigen könnte man sagen, daß diejenige Arbeit notwendig ist, mit der sie ihre jeweiligen Einkommens- bzw. Konsumansprüche befriedigen können. Sodann aus der Sicht kapitalistischer Unternehmen erscheint ein Einsatz von Lohnarbeit sinnvoll und notwendig, wenn und insoweit durch ihn eine profitable Kapitalakkumulation organisiert werden kann. Und schließlich gibt es noch die Sichtweise des kulturkritischen Beobachters, der die Auffassung vertritt, daß ein Großteil dessen, was heute produziert wird bzw. in Zukunft noch produziert werden kann, keineswegs sinnvoll ist und daß deshalb auch die in dieser Produktion eingesetzte Arbeit keineswegs notwendig ist. Wenn ich Gorz richtig verstanden habe, nimmt er die zuletzt genannte Betrachtungsweise ein. Dann aber ist es offenbar wichtig, folgende Behauptungen zu unterscheiden: daß ein zunehmend großer Teil der gegenwärtigen und in Zukunft noch möglichen Produktion nicht sinnvoll und notwendig ist; und daß es langfristig zu einer immer mehr abnehmenden Nachfrage nach Arbeit kommen wird.

Ganz ähnlich verhält es sich mit der Behauptung, daß technischer Fortschritt ein wesentlich arbeitsparender Prozeß ist. Demn um die Vorstellung bilden zu können, daß technischer Fortschritt Arbeit einspart, muß man ein Produktionsziel voraussetzen, an dem der erforderliche Arbeitsaufwand gemessen werden kann. Die Frage, ob durch technischen Fortschritt Arbeit immer weniger »notwendig « wird, läßt sich nur willkürlich von der Frage trennen, wofür und für wen Arbeit überhaupt »notwendig« ist. Man erkennt das etwas genauer, wenn man wiederum zwei Fragen unterscheidet: Erstens, welche Rolle der technische Fortschritt in der Dynamik einer kapitalistischen Ökonomie tatsächlich spielt; und zweitens, worin man einen Sinn des technischen Fortschritts sehen kann. Gorz bezieht seine Überlegungen hauptsächlich aus dieser zweiten Fragestellung, und eine seiner zentralen Behauptungen lautet dementsprechend: der Sinn des technischen Fortschritts liegt darin, bisher notwendige Arbeiten überflüssig zu machen. So sagt er zum Beispiel: Man kann von der Technik »verlangen, die Effektivität der Arbeit zu steigern und ihre Dauer, ihre Mühsal zu mindern. Aber man muß wissen, daß die gewachsene Macht der Technik ihren Preis hat: sie entzweit die Arbeit vom Leben (...) Dieser Preis für die Technisierung wird nur in dem Ausmaß akzeptabel, wie diese Arbeit und Zeit einspart: Dies ist ihr erklärtes Ziel - ein anderes hat sie nicht. Die Technik ist dafür da, die Menschen mehr und besser mit weniger Anstrengung und in kürzerer Zeit produzieren zu lassen. « (S. 131) Aber dies ist offensichtlich eine Sinndeutung des technischen Fortschritts, über die man unterschiedlicher Meinung sein kann. Denn man könnte mit zunächst gleicher Berechtigung behaupten, daß der Sinn des technischen Fortschritts nicht darin liegt, ein vorgegebenes Spektrum an Bedürfnissen mit immer weniger Arbeitsaufwand befriedigbar zu machen, sondern darin, die Menschen in die Lage zu versetzen, die vorgefundene Natur in immer größerem Maße durch eine ausgedachte und konstruierte künstliche Welt, also durch eine menschliche im Unterschied zu einer natürlichen Welt, zu ersetzen, und dadurch menschlicher Selbstverwirklichung zu dienen; und dann wären Grenzen dafür, menschlicher Arbeit durch technischen Fortschritt einen Sinn geben zu können, grundsätzlich unabsehbar. 
Die zunächst entscheidende Frage ist jedoch gar nicht, welche Sinndeutung des technischen Fortschritts »wir uns zu eigen machen, sondern welche Rolle der technische Fortschritt in der Dynamik einer kapitalistischen Ökonomie tatsächlich spielt. Vermutlich nicht die, die wir uns wünschen. Aber daraus folgt zugleich, daß wir aus unseren Sinndeutungen des technischen Fortschritts nicht unmittelbar Schlußfolgerungen im Hinblick auf seine tatsächliche Bedeutung in der Entwicklung einer kapitalistischen Ökonomie ziehen können. Sobald man jedoch versucht, die Behauptung eines langfristig arbeitsparenden technischen Fortschritts nicht nur als subjektive Sinndeutung zu begründen, müßte zweierlei gezeigt werden: Erstens, daß kapitalistische Unternehmen ein Interesse daran haben, möglichst wenig Arbeitskraft auszubeuten; und zweitens, daß daraus auch eine Tendenz des ökonomischen Gesamtsystems resultiert, mit möglichst wenig Arbeitseinsatz auszukommen. Beides kann aber für eine kapitalistische Ökonomie sicherlich nicht begründet werdell. In ihrer makroökonomischen Dynamik ist sie ein Prozeß, in dem fortwährend - vor allem auch durch technischen Fortschritt - neue Produktionsmöglichkeiten erschlossen werden und bisherige Verwendungsweisen von Arbeit obsolet werden. ${ }^{3}$ Ob dieser Entwicklungsprozeß langfristig immer weniger Arbeit notwendig macht, ist dabei eine durchaus offene Frage und sicherlich auch davon abhängig, von welchen Sinndeutungen dieses Prozesses sich die beteiligten Akteure leiten lassen. Gorz stellt sich jedoch, auch in diesem Fall, gar nicht die Frage, wie sich seine Sinndeutung des technischen Fortschritts zu denjenigen Sinndeutungen verhält, die die Enttscheidungsprozesse anleiten, in denen in einer kapitalistischen Ökonomie über die Entwicklung und den Einsatz technischen Fortschritts entschieden wird. Schon deshalb kann auf diese Weise nicht die Erwartung begründet werden, daß es zu einer langfristig sinkenden Nachfrage nach Arbeit kommen wird.

\section{Gibt es Grenzen einer kapitalistischen Ökonomie?}

Gorz' Versuch, ökonomische Rationalisierung als einen arbeitsparenden Prozeß zu interpretieren, kann also als eine Technikphilosophie in normativer Absicht verstanden werden; sie liefert jedoch keine unmittelbare Antwort auf die Frage, in welchem Umfang im Rahmen einer kapitalistischen Ökonomie langfristig Arbeitsplätze organisiert werden können, wie die langfristige Arbeitsmarktentwicklung insbesondere auch von Ideologiebildungen abhängt, die den beteiligten Akteuren Sinnkriterien für ihre Entscheidungen vermitteln. Um so wichtiger erscheint deshalb ein zweiter Gedankengang, mit dem Gorz Grenzen ökonomischer Rationalisierung aufzuzeigen versucht. Gorz will zeigen, daß sich keineswegs alle menschlichen Tätigkeiten dafür eignen, einem Proze $\beta$ ökonomischer Rationalisierung unterworfen zu werden, so daß infolgedessen der Expansionsprozeß einer kapitalistischen Ökonomie - insofern er wesentlich auch darin besteht, stets neue Tätigkeiten als warenproduzierende Lohnarbeiten kapitalistisch zu organisieren - auf Grenzen stoßen muß. Der Zusammenhang mit der Vermutung eines beginnenden Endes der Arbeitsgesellschaft ergibt sich 
insbesondere aus folgender Überlegung: daß der Prozeß ökonomischer Rationalisierung nur bei solchen Tätigkeiten erfolgreich durchgeführt werden kann, die sich »effizient«, mit der Zielsetzung einer Produktivitätssteigerung, organisieren lassen und bei denen mithin ein arbeitsparender technischer Fortschritt stattfinden kann. ${ }^{4}$

Gorz entwickelt seinen Gedankengang so, daß er zunächst vier Kriterien formuliert und dann zu zeigen versucht, daß nur solche Tätigkeiten einem Prozeß ökonomischer Rationalisierung unterworfen werden können, die diesen Kriterien genügen. Wie er ausführt, muß es sich um Tätigkeiten handeln, »die a) einen Gebrauchswert schaffen; b) dies zum Zwecke des Erwerbs tun; c) in der öffentlichen Sphäre verrichtet werden; sowie d) zeitlich und im Hinblick auf größtmögliche Leistung gemessen werden können.«(S. 197) Und er fügt hinzu: »Im Gegensatz zu einer weitverbreiteten Auffassung reicht also der Umstand, daß eine Tätigkeit zu Erwerbszwecken (d.h. für Bezahlung) ausgeübt wird, nicht aus, um diese bereits als Arbeit im ökonomischen Sinne ansehen zu können.« Damit soll vermutlich ausgesagt werden, daß nur »Arbeiten im ökonomischen Sinne «, d.h. Arbeiten, die den genannten vier Kriterien genügen, kapitalistisch organisiert werden können. ${ }^{5}$ Es stellen sich also zwei zusammenhängende Fragen: Erstens, was mit diesen Kriterien gemeint ist; und zweitens, ob durch sie wirklich notwendige Bedingungen ökonomischer Rationalisierung - insbesondere: einer Verwandlung menschlichen Lebens in kapitalistisch organisierte Lohnarbeit und Konsum kapitalistisch produzierter Güter und Dienstleistungen - formuliert werden bzw. welcher Art die Grenzen sind, die durch sie formuliert werden sollen.

a) Setzt ökonomische Rationalisierung voraus, daß Gebrauchswerte geschaffen werden? Das ist sicherlich nicht strittig, wenn darunter folgendes verstanden wird: einer Tätigkeit muß eine monetär bewertbare Leistung zurechenbar sein, die von einer anderen Per'son angeeignet werden kann. Diese Formulierung macht allerdings zugleich deutlich, daß es nicht vom »Charakter« einer Tätigkeit abhängt, ob sie einen Gebrauchswert erzeugt oder nicht, daß entscheidend dafür vielmehr die Herausbildung eines Interesses bei denjenigen ist, die als potentielle Käufer von Arbeitskraft oder ihrer Produkte in Erscheinung treten und sich durch einen Kauf von Arbeitskraft zugleich ein (umstrittenes) Recht erwerben, den »Charakter« der von ihr zu leistenden Arbeit zu definieren. Wenn es hier eine Grenze gibt, dann kann sie jedenfalls nicht darin bestehen, daß einige Tätigkeiten keine Gebrauchswerte erzeugen (was selbstverständlich richtig ist); sondern es müßte gezeigt werden, daß es Grenzen dafür gibt, aus der Verfügung über die Arbeitskraft anderer Menschen bzw. die von ihr produzierten Gebrauchswerte einen Nutzen ziehen zu können. Aber gibt es solche Grenzen? Ich stimme Gorz zu, daß solche Grenzen nur als »Sinngrenzen« thematisiert werden können; d.h. diese Grenzen liegen dort, wo die potentiellen Nutznießer fremder Arbeitskraft bzw. ihrer Produkte darin keinen Sinn mehr sehen können. In gewisser Weise impliziert diese Betrachtungsweise jedoch, daß es infolgedessen keine »festen Grenzen« gibt, sondern daß der Bildungsprozeß von Kriterien für den Sinn der Nutzung fremder Arbeitskraft bzw. ihrer Produkte als prinzipiell offen und kontingent angesehen werden muß. Dies wiederum heißt zwar nicht, daß sich Sinngrenzen - oder explizit normative Grenzen ${ }^{6}$ - nicht mehr formulieren lassen; wohl aber, daß ihre For- 
mulierung als ein grundsätzlich unsicherer, in seinem Erfolg nicht kalkulierbarer Versuch der Einflußnahme auf einen kontingenten Prozeß der Herausbildung von Sinnkriterien anzusehen ist.

b) Setzt ökonomische Rationalisierung einen Erwerbszweck voraus? Faßt man den Begriff »Erwerbszweck «hinreichend allgemein, kann auch diese Frage sicherlich bejaht werden; aber auch in diesem Fall folgen daraus nicht notwendigerweise Grenzen ökonomischer Rationalisierung. Denn man kann sich zwar vorstellen, daß in der Folge eines zunehmenden gesellschaftlichen Wohlstands das Erwerbsmotiv bei denjenigen an Bedeutung verliert, die es nur nach Maßgabe einer von ihnen dafür einzusetzenden Arbeits- und Lebenskraft verfolgen können. Es ist jedoch schwer vorstellbar, daß das Erwerbsmotiv auch dort sinnlos werden könnte, wo es durch die Ausbeutung fremder Arbeitskraft verfolgt werden kann. Jedenfalls bisher hat sich die von Sombart, Schumpeter und anderen formulierte These, daß der $»$ Geist des Kapitalismus« langfristig seine Motivationskraft verlieren wird, nicht bestätigt. Also wäre genauer zu überlegen, ob eine kapitalistische Ökonomie langfristig deshalb an Grenzen stoßen muß, weil immer weniger Menschen bereit sein werden, sich ihr als Lohnarbeiter anzubieten. Gorz reflektiert diese Frage fast ausschließlich mit ideologiekritischen Erwägungen: er kritisiert die Erwerbstätigen wegen ihrer »Arbeitsbesessenheit« und ihrer sich fortwälhrend steigernden Konsumansprüche. Aber er verfehlt damit ein wesentliches Merkmal ökonomischer Rationalisierung: daß durch eine Verwandlung von Lebenstätigkeiten in kapitalistisch organisierte Lohnarbeit und Konsum kapitalistisch produzierter Güter und Dienstleistungen Lohnarbeit tatsächlich immer notwendiger wird, um den gesellschaftlichen Lebensbedingungen entsprechend leben zu können; oder anders formuliert: daß durcl den Prozeß kapitalistischer Rationalisierung menschliches Leben zunehmend tatsächlich zu einem Prozeß des Arbeitens und des Konsumierens wird und infolgedessen auch zunehmend aus diesen Lebensformen seinen Sinn gewinnen muß. Ich stimme Gorz zwar in der Fragestellung zu, ob und wie alternative Lebenschancen und Lebensformen vorstellbar gemacht und realisiert werden können. Aber um eine Antwort finden zu können, sollte man berïcksichtigen, daß der Erfolg kapitalistischer Rationalisierung ebenfalls darauf beruht: stets neue Lebenschancen und Lebensformen vorstellbar und realisierbar zu machen, wobei deren attraktive Vielfalt jedenfalls bisher noch auch vom Ausmaß der Ungleichheit in der Einkommens- und Vermögensverteilumg abhängt.

c) Verlangt ökonomische Rationalisierung, daß die von ihr organisierten Tätigkeiten in einer »öffentlichen Sphäre« stattfinden? Verwendet man diesen Begriff in seiner gewöhnlichen Bedeutung, ist das sicherlich nicht der Fall. Lohnarbeit findet vielmehr typischerweise in »privaten« Räumen statt (als Fabrikarbeit, als Heimarbeit usw.), in Räumen, die durch partikulare Interessen organisiert werden. ${ }^{7}$ Es ist jedoch nicht vollständig klar, was Gorz mit diesem Kriterium aussagen will. Eine Interpretationsmöglichkeit ergibt sich vielleicht aus folgender Erläuterung:

»Die Tatsache, daß eine Tätigkeit in der Öffentlichkeit zu Erwerbszwecken ausgeübt wird, signalisiert gleichzeitig, daß es sich um eine geselIschaftlich nützliche, einen Gebrauchswert schaffende und als solche anerkannte Tätigkeit handelt. Mit anderen Worten gehört diese Tätigkeit zu einem >Beruf $\varsigma$ : sie hat ei- 
nen öffentlichen Preis und Sozialstatus, ich kann sie mir von einer beliebigen Anzahl von Kunden oder Arbeitgebern bezahlen lassen, ohne mit diesen eine persönliche oder private Beziehung pflegen zu müssen.« (S. 198)

Auch mit dieser Beschreibung wird jedoch keine notwendige Bedingung ökonomischer Rationalisierung formuliert. Sie mag zwar in vielen Fällen angemessen sein, aber jeder kennt auch genügend Fälle, in denen sie nicht zutrifft, in denen ein (Lohn-) Arbeitsverhältnis durch persönliche und private Beziehungen geprägt wird, die Form eines mehr oder weniger weitgehenden Zwangsverhältnisses annimmt, und gleichwohl ökonomischer Rationalisierung unterworfen wird. Ich stimme also Gorz nicht $\mathrm{zu}$, wenn er schreibt: »Wenn private Bande bestehen, schließen sich die käuflichen Beziehungen aus.« (S. 200) Man kann dies vielleicht als ein normatives Urteil akzeptieren: die Menschen sollten ihre privaten Beziehungen nicht auf der Grundlage monetär vermittelter Kosten-Nutzen-Kalküle organisieren. Aber welche ihrer Beziehungen sind »privat «? In Wirklichkeit wurden und werden die von Gorz formulierten Sinnvoraussetzungen bzw. Sinngrenzen ökonomischer Rationalisierung fortwährend durchbrochen und verändern sich dadurch zugleich auch die Grenzen und die Verfaßtheit dessen, was Gorz die »privaten « Beziehungen der Menschen untereinander nennt. Ein besonders wichtiges Beispiel ist in diesem Zusammenhang die hauptsächlich von Frauen verrichtete Hausarbeit. Gorz bemüht sich zu zeigen, daß die in diesem Begriff zusammengefaßten Tätigkeiten nicht ökonomisch rationalisiert, insbesondere nicht in der Form von Lohnarbeit kapitalistisch organisiert werden können, da sie andernfalls ihren herkömmlichen Sinn (und Gebrauchswert) verlieren würden. Tatsächlich ist dieser Prozeß jedoch schon weit vorangeschritten, handelt es sich bei einem erheblichen Teil der heutigen typischen »Frauenberufe« um Tätigkeiten, die vorher in häuslichen Lebensgemeinschaften verrichtet wurden; und ein Ende dieses Prozesses ist kaum absehbar. Natürlich verändert sich infolgedessen der »Charakter « dieser 'Tätigkeiten und ändern sich auch die Formen des Zusammenlebens der Menschen. Aber das beweist zunächst nur, daß aus dem »Charakter « einer Tätigkeit (und aus den jeweils vorhandenen Institutionen des menschlichen Zusammenlebens) keine zwingenden Grenzen ihrer ökonomischen Rationalisierung resultieren, und daß also zu unterscheiden ist: ob ein Prozeß ökonomischer Rationalisierung stattfinden kann und ob und wie er stattfinden sollte. ${ }^{8}$

d) Schließlich bleibt noch zu überlegen, ob ökonomische Rationalisierung nur stattfinden kann, wenn Arbeitszusammenhänge »zeitlich und im Hinblick auf größtmögliche Leistung gemessen werden können «. In gewisser Weise folgt dies unmittelbar aus dem Begriff ökonomischer Rationalisierung, denn sie intendiert per definitionem ein möglichst optimales Kosten-Nutzen-Verhältnis. Auch in diesem Fall muß aber gefragt werden, ob sich daraus irgendwelche Grenzen ökonomischer Rationalisierung ergeben. Ich glaube nicht, daß das der Fall ist.

Gorz scheint zu meinen, daß eine Tätigkeit nur dann als (Lohn-)Arbeit organisiert und ausgebeutet werden kann, wenn es möglich ist, ihr in irgendeinem Sinne des Wortes eine »physische Produktivität « so zuzurechnen, daß sie gesteigert werden kann. Aber ich glaube, daß dieser Gedanke abstrakter gefaßt werden muß, damit er nicht irre- 
führend wird. Ökonomische Rationalisierung impliziert das Bestreben, ein möglichst optimales Kosten-Nutzen-Verhältnis zu erreichen. Dies kann im allgemeinen auf zweierlei Weisen versucht werden. Erstens durch eine Einflußnahme auf die monetären Relationen (Kosten und Erlöse); zweitens durch Veränderungen in der technischen und organisatorischen Verfassung der jeweiligen Arbeitsprozesse. Ob und inwieweit solche Veränderungen der Arbeitsprozesse mit dem Ziel, ein besseres Kosten-Nutzen-Verhältnis zu erreichen, möglich sind, hängt sicherlich zum Teil vom »Charakter « dieser Arbeitsprozesse ab; aber eben nur zum Teil. Denn dieser »Charakter « der Arbeitsprozesse ist keineswegs ein dem Prozeß ökonomischer Rationalisierung fest vorgegebener Sachverhalt, sondern er kann sich - gerade infolge ökonomischer Rationalisierung - sehr weitgehend verändern. Ein deutliches Beispiel dafür, das auch von Gorz angeführt wird, bilden die Veränderungen im »Charakter« der Behandlungs- und Pflegetätigkeiten in Krankenhäusern, die eine Folge ihrer zunehmenden ökonomischen Rationalisierung sind. Gorz interpretiert dieses Beispiel so, daß in diesen Veränderungen Grenzen ökonomischer Rationalisierung sichtbar werden. Er beruft sich dafür auf den Sinn, den die sich verändernden Tätigkeiten »eigentlich« haben. Das entscheidende Problem besteht jedoch darin, daß dieses Beispiel (wie viele andere) zeigt, daß es diese von Gorz formulierten Grenzen nicht gibt, daß es sich um subjektive Sinnvoraussetzungen - und in analytischer Rekonstruktion also um normative Urteile - handelt, über die sich der Prozeß ökonomischer Rationalisierung offensichtlich hinwegsetzen kann. ${ }^{9}$

Zusammenfassend läßt sich sagen, daß alle vier Kriterien, die Gorz formuliert, auf mögliche Sinngrenzen ökonomischer Rationalisierung verweisen, daß jedoch darin zugleich ihre Problematik liegt. Denn in Wirklichkeit wurden und werden die von Gorz formulierten Sinnvoraussetzungen bzw. Sinngrenzen ökonomischer Rationalisierung fortwährend durchbrochen, so daß es irreführend ist, hier von »ontologischen, existentiellen Grenzen der Rationalisierung « zu sprechen. Um das Problem deutlich zu machen, könnte man sogar sagen, daß es zum Wesen des Prozesses ökonomischer Rationalisierung gehört, herkömmliche, durch Traditionen vermittelte Sinngrenzen zu durchbrechen, neue Nutzungsmöglichkeiten der Arbeitskraft zu erschließen und durch ein Angebot neuer Produkte und Dienstleistungen zugleich neue Chancen für subjektive Sinndeutungen - also neue Lebenschancen - a11zubieten. Natürlich war und ist dieser Prozeß stets umstritten, sowohl im Hinblick auf seine materiellen Folgen als auch in seiner Sinnhaftigkeit; und wenn Gorz sagt, daß eine Nichtbeachtung der von ihm formulierten Sinngrenzen zu »vernunftwidrigen Pseudo-Rationalisierungen « führt, kann man dies insoweit als eine Stellungnahme zu solchen Auseinandersetzungen anerkennen. Aber ein besonders wichtiges Problem, um das sich Gorz nicht kümmert, besteht infolgedessen darin, wie eine solche Kritik ökonomischer Rationalisierung überhaupt zur Geltung gebracht werden kann, oder anders gesagt: wie diese Auseinandersetzungen selbst rationalisiert werden können. 


\section{Wie ist Kritik ökonomischer Rationalität möglich?}

Sinnvoraussetzungen bzw. Sinngrenzen ökonomischer Rationalisierung können nur Geltung erlangen oder bewahren, wenn die an den gesellschaftlichen Produktionsund Konsumtionsprozessen beteiligten Akteure sie für bzw. durch ihre Handlungsweisen anerkennen, sei es durch Einsicht oder durch äußerlich (rechtlich) verbindlich gemachte Einschränkungen ihrer Handlungsfreiheit. Die etwas irreführende Fragestellung, ob es Grenzen ökonomischer Rationalisierung gibt, sollte deshalb zunächst so reformuliert werden: ob und wie ökonomischer Rationalisierung Grenzen gesetzt werden könnell. Um auf diese Frage eine Antwort zu finden, erscheint es vor allem wichtig, genauer zu verstehen, wie sich der Prozeß ökonomischer Rationalisierung vollzieht und durchsetzt. Diese Aufgabe wird auch von Gorz betont: daß wir »begreifen (müssen), worin die ökonomische Rationalität besteht und worin die innerste, motivationale Triebkraft jenes Imperialismus besteht, den sie gegenüber anderen Rationalitätstypen an den Tag legt. Warum traf die >Kolonialisierung der Lebenswelt nicht bereits früher auf stärkeren Widerstand? Warum konnte das >Muster der kapitalistischen Modernisierung $<$ Fuß fassen und eine >unaufhaltsame Eigendynamik der ökonomischen und administrativen Subsysteme entfalten? Welche motivationalen Grundlagen gibt es innerhalb der ökonomischen Rationalität. die es ihr erlaubt haben, im Leben der Individuen Platz zu greifen und spontane, affektive, solidarische Beziehungen zu verdrängen? «(S. $154 \mathrm{f}$.)

Gorz' Versuch, auf diese Fragen eine Antwort zu finden, ist zwar schwer nachvollzielıbar; man findet aber vielleicht einen Zugang, wenn man seine Ausführungen als einen Versuch ansieht, zu einem tieferen Verständnis und einer besseren Begründung des gängigen kulturkritischen, ideologiekritischen und funktionalistischen Geredes über die lebensfeindliche Maßlosigkeit des Kapitalismus zu gelangen. Das ist natürlich nicht einfach, wie Gorz selbst feststellt:

»Der Leser wird bemerkt haben, daß die Analysen bisher unaufhörlich zwischen zwei Ebenen hin und her gegangen sind: das Wachstum erschien bald als Bestreben der Individuen, bald als Erfordernis der Einzelkapitale, bald als makro-ökonomische Notwendigkeit für das (kapitalistische) System. Das liegt daran, daß die Erfordernisse auf diesen verschiedenen Ebenen eine nahezu perfekte Übereinstimmung untereinander aufweisen. Es genïgt, die Quantifizierung als Bewertungsmethode und Entscheidungskriterium zu übernehmen, damit auf allen Ebenen das Streben nach unbegrenztem Wachstum entsteht: als Unzufriedenheit, Lust und Gier auf >mehr< auf der Ebene der Individuen; als Elfordernis unbegrenzter Maximie-rung auf der Ebene der Einzelkapitale; als Erfordernis beständigen Wachstums auf der Ebene des Systems; als ideologische Bewertung von Höchstleistungen (Geschwindigkeit, Maschinenkraft, Höhe der Gebäude, landwirtschaftlicher Ertrag usw.) auf der Ebene der Zivilisation.« (S. 174 f.)

Wie dieses Zitat bereits andeutet, versucht Gorz, zu einem tieferen Verständnis zu gelangen, indem er ökonomische Rationalisierung als einen Prozeß der Herrschaft ökonomischer Rationalität über die Menschen und ihre Ökonomie deutet. Das Wesen dieses Prozesses sieht er darin, daß ein Denken in monetär vermittelten KostenNutzen-Kalkülen von den Menschen gewissermaßen Besitz ergriffen und sie dadurch enteignet habe. Er beschreibt deshalb dieses Denken als formal, abstrakt, rechenhaft, quantifizierend, als »Prototyp der verdinglichen Rationalisierung« (S. 157), kurz 
gesagt als einen schroffen Gegensatz zu allem menschlich-lebendigen Selbstsein. Dadurch, daß die Menschen von der Krankheit solchen Denkens befallen und überwältigt worden sind, seien sie maßlos und (deshalb?) zugleich Sklaven dieses Denkens geworden, entscheiden sie nicht mehr selbst über ihre Handlungen, sondern entscheidet nun dieses Denken - die ökonomische Rationalität - über sie und ihr Leben.

So heißt es etwa: »Die ökonomische Rationalität beginnt mit dem rechnerischen Kalkül.« (S. 156) »Einsatz und Sinn der >ökonomisch rationellen< Entscheidung sind jeder Möglichkeit rationaler Überprüfung bereits dadurch entzogen, daß die ökonomische Rationalität selbst in Rechenverfahren und -formeln formalisiert ist, die ihrerseits der Diskussion und Reflexion entzogen bleiben.« (S. 177 f.) »Der Imperialismus der >kognitiv-instrumentellen Vernunft und insbesondere der ökonomischen Rationalität hängt - auf der Ebene des Subjekts - von der scheinbaren Objektivität der Urteilskriterien ab, die der mathematische Kalkül liefert. Dieser ist ja, wie wir sahen, eine Technik, die das Subjekt davon enthebt, der jeweiligen Entscheidung einen Sinn zu verleihen und sie als eigene Entscheidung zu übernehmen: Der Kalkül wird von selbst entscheiden.« (S. 182) Und zwar maßlose Entscheidungen, denn: »Indem sie alles quantifizierte, um alles berechenbar zu machen, verniclıtete die ökonomische Rationalisierung sonit jedes Kriterium, das es ermöglichte, sich zufrieden zu geben mit dem, was man hatte, was man gemacht hatte oder sich zu tun volnahm. « (S. 163) Und schließlich zwingt der Kapitalismus unterschiedslos allen seine maßlose Rationalität auf: »Es genügt, die Quantifizierung als Bewertungsmethode und Entscheidungskriterium zu übernehmen, damit auf allen Ebenen das Streben nach unbegrenztem Wachstum entsteht.« (S. 174) Oder so: »Was im >Geist des Kapitalismus« aus der Leidenschaft des Organisierens und Quantifizierens entsprang, das mußte bei den Konsumenten der >Überflußgesellschaft ‘ aus einer `Mimesis $<$ entspringen: d.h. aus dem - übrigens durch die kommerzielle Werbung methodisch inszenierten - Verlangen, all das auch zu haben, was $>$ die anderen $\times$ haben, ob es nun mehr, besser oder einfach etwas anderes ist als das, was man selber hat.« (S, $165 \mathrm{f}$.)

Wie in diesen und vielen ähnlichen Ausführungen seines Buches deutlich wird, verknüpft Gorz zweierlei: eine bestimmte Beschreibung ökonomischer Rationalität (die sich schließlich in einen abstrakten Gegensatz kalkulierender Rationalität und nichtentfremdeten Lebens auflöst) mit der Annahme, daß eine solche Rationalität die Herrschaft über die Menschen und ihre Ökonomie gewonnen habe. Diese Verknüpfung macht es ihm möglich, seine Vorstellung einer Herrschaft ökonomischer Rationalität dann auch zur Formulierung von Bezugsproblemen für funktionalistische und ideologie-kritische Deutungen realer Interaktionszusammenhänge zu verwenden.

Seine funktionalistischen Deutungen folgen stets etwa diesem Muster: Weil die Menschen von der Idee ökonomischer Rationalität beherrscht werden, sind sie gezwungen, dieser Idee zu gehorchen; sie haben zwar ihre gesellschaftlichen Verhältnisse zweckmäßig eingerichtet, aber zweckmäßig eben nur für die Zwecke der ökonomischen Vernunft selbst. Und ganz analog (und ganz im Denkhorizont der gängigen Kulturkritik) formuliert Gorz auch seine Ideologiekritik an denjenigen, die sich, wie er sagt, als Erwerbstätige und Konsumenten in den Dienst der ökonomischen Vernunft stellen. Er kritisiert die »Arbeitsbesessenheit « der Erwerbstätigen, die nicht begreifen wollen, daß ihre Arbeit zunehmend sinnlos wird; und er kritisiert die Konsumenten, die fortwährend ihre Ansprüche steigern (oder aus Gorz'scher Sicht besser formuliert: die nicht selbst ihre Ansprüche steigern, sondern zulassen, daß ihre Ansprüche im Namen ökonomischer Rationalität stets gesteigert werden) und die dadurch zu entfremdeten Funktionären des ökonomischen Wachstums- und Rationalisierungsprozesses werden. So sagt er zum Beispiel, »daß die Arbeits- und Verdienstbesessenheit beim 
Arbeiter denselben Sinn hat wie die Leidenschaft des Rechenkalküls für die Entstehung des Kapitalismus: Die Arbeit diszipliniert und ordnet das Leben, sie schützt das Individuum vor dem Ruin der normativen Gewißheiten und vor der Verpflichtung, sein Leben in die eigenen Hände zu nehmen. «(S. 171) Aber vor allem richtet sich die Gorz'sche Kritik dagegen, daß es der kapitalistischen Ökonomie gelingt, stets neue Konsum- und damit Lebens- und Entfaltungschancen für die Mitglieder der Gesellschaft zu schaffen. »Entscheidend ist, daß der $>$ Geist des Kapitalismus $\prec$ das Band zwischen Arbeit und Bedürfnis zerschnitt.«(S. 162) Denn: »In dem Maße, wie der Konsum die Grenze der tatsächlich empfundenen Bedüıfnisse überschreitet, kann er in den Dienst der Produktion, d.h. der `Bedürfnisse < des Kapitals gestellt werden. Hier liegt das Geheimnis der > unaufhaltsamen Eigendynamik<, mit der das >ökonomische Subsystem< seinen Einflußbereich beständig ausweitet.«(S. 173)

Wenn ich Gorz richtig verstehe, möchte er mit seinen Ausführungen erreichen, daß die Menschen, indem sie anerkennen, wie sie von Gorz beschrieben werden - nämlich als beherrscht durch ökonomische Rationalität -, dadurch zur Besinnung kommen, wieder ihre eigenen Bedürfnisse, ihr eigenes Leben, sich selbst entdecken und gegen die Herrschaft ökonomischer Rationalität zur Geltung birngen. Man muß sich jedoch überlegen, ob das Ziel, ökonomischer Rationalisierung Grenzen zu setzen, auf diese Weise erreicht werden kann. Denn Gorz' Kritik richtet sich, wie deutlich geworden sein sollte, im wesentlichen gegen die subjektive Verfassung der Menschen, die sich als Erwerbstätige und Konsumenten Lebenschancen zu erschließen versuchen, so daß ihnen im Namen dieser Kritik doch auch noch ihre Empfänglichkeit für deren Botschaft abgesprochen werden könnte. Oder etwas anders formuliert: Gorz verlegt gewissermaßen den Ort der $»$ Herrschaft des Kapitals« in die Innerlichkeit der Beherrschten, in ihr Denken, aus dem heraus dann der Prozeß ökonomischer Rationalisierung seinen stets erneuten Siegeszug antreten kann. Infolgedessen wird es aber unklar, was dann überhaupt noch kritisiert werden kann. Denn wenn Gorz recht hätte, entsprächen die durch den Prozeß ökononischer Rationalisierung erschlossenen Lebenschancen, Arbeits- und Konsummöglichkeiten doch ziemlich genau den Wünschen und Bedüifnissen der Akteure dieses Prozesses?

\section{Schlußbemerkungen}

Gorz' Kritik ökonomischer Rationalität bzw. Rationalisierung läuft darauf hinaus, die beteiligten Akteure zu kritisieren, weil sie sich in ihrem Arbeits- und Konsumverhalten von »falschen« Sinnkriterien leiten lassen. Solche Kritik sollte sicherlich ernstgenommen werden; aber zugleich sollte auch gefragt werden, was mit ihr erreicht werden kann. Mein hauptsächlicher Einwand verdankt sich dieser Fragestellung: daß Gorz die Aufgaben einer Kapitalismuskritik zu einer Ideologiekritik an den beteiligten individuellen Akteuren verkürzt. Den wichtigsten Grund dafür sehe ich darin, daß Gorz es versäumt, sich mit dem Problem sozialer Macht kapitalistischer Unternehmen auseinander zu setzen. Er legt uns ein Bild nahe, bei dem kapitalistische Unternehmen 
gewissermaßen als durch ökonomische Rationalität, durch ein Prinzip der Profitmaximierung gesteuerte Produktionsmaschinen erscheinen. Wäre dies eine zutreffende Beschreibung, könıte man annehmen, daß die ökonomische Entwicklung im wesentlichen vom Arbeits- und Konsumverhalten ilırer Akteure - davon, welchen Gebrauch sie von der kapitalistischen Produktionsmaschine machen - abhängig ist. Ich möchte jedoch einwenden, daß es sich nicht um eine zutreffende Beschreibung handelt, daß eine angemessene Beschreibung kapitalistische Unternehmen vor allem auch als Organisatoren sozialer Macht sichtbar machen müßte, d.h. als Organisationen, die durch ihre Entscheidungen wesentlich dazu beitragen, die von den Mitgliedern der Gesellschaft individuell wahrnehmbaren Handlungschancen (Lebens-, Arbeits- und Konsumchancen) zu bestimmen.

Die Konsequenz wäre eine weitgehend andere gesellschaftspolitische Problemsicht. Das wesentliche Problem bestünde damn nicht darin, das Streben nach Erwerbs- und Konsumchancen zu kritisieren, sondern in einem Mangel an individuellen und politischen Handlungschancen, um gegen die soziale Macht kapitalistischer Unternehmen - und ihren Anspruch, die gesellschaftlichen Lebensverhältnisse ihren Sinnkriterien entsprechend zu gestalten --eigene, altemative Sinnkriterien durchsetzen zu können. Das wesentliche Problem bestünde dann also in der institutionellen Verfassung der Ökonomie, die es den kapitalistischen Unternehmen ermöglicht, ihre soziale Macht weitgehend ungehindert zu entfalten und damit zugleich die Bedingungen zu bestimmen, unter denen die Mitglieder der Gesellschaft ihre Lebenschancen gewinnen müssen.

Begrüıdung und Implikationen einer solchen anderen Problemsicht können hier leider nicht näher ausgeführt werden. Es sollte jedoch deutlich geworden sein, daßes folgenreich ist, wie man den Prozeß ökonomischer Rationalisierung beschreibt; denn je nachdem kommt man zu unterschiedlichen Schlußfolgerungen im Hinblick auf die Aufgabe, diesem Prozeß Grenzen zu setzen. Gorz begründet seine primäre Forderung nach einer langfristig zunehmenden Arbeitszeitverkürzung vor allem damit, daß die Erwerbstätigen dadurch Zeit gewinnen können, um sich von der Herrschaft ökonomischer Rationalität zu befreien. Zum Beispiel: »Die Arbeiter werden die Grenzen der ökonomischen Rationalität nur dann entdecken können, wenn ihr Leben nicht völlig durch die Arbeit besetzt und ihr Geist nicht völlig von ihr beherrscht wird.« (S. 168) Begreift man dagegen ökonomische Rationalisierung auch als einen Prozeß sozialer Machtentfaltung kapitalistischer Unternehmen, wird zwar die Forderung nach Arbeitszeitverkürzung nicht falsch, erweist sie sich aber als wesentlich unzureichend, um der ökonomischeı Rationalisierung Grenzen setzen zu könıen. Denn sie greift die soziale Macht kapitalistischer Unternehmen im Kern nicht an (insoweit ganz ähnlich wie die weitgehende Beschränkung gewerkschaftlicher Kämpfe auf Verteilungsauseinandersetzungen). Um ökonomischer Rationalisierung Grenzen zu setzen, müßte man vielmehr zumindest auf einen zentralen Gedanken der sozialistischen Tradition zuriickkommen: daß die soziale Macht kapitalistischer Unternehmen einer politischen Kontrolle durch die Mitglieder der Gesellschaft unterworfen werden muß. Gorz' Vorschläge zurArbeitszeitverkürzung und zur Gewährleistung von Einkom- 
mensansprüchen für alle Mitglieder der Gesellschaft sollten also auch daraufhin diskutiert werden, ob und wie sie zu einer Lösung dieser Aufgabe beitragen können.

\section{Anmerkungen}

1 Das Buch erscheint (als Übersetzung aus dem Französischen von Otto Kallscheuer) im Rotbuch-Verlag (Berlin). Seitenangaben hinter den Zitaten aus diesem Buch beziehen sich auf die Druckfahnen; das eingangs angefühtte Zitat: S. 257.

2 Es ist deshalb etwas irreführend, wenn Gor'z seine These als Formulierung einer » Tatsache « darstellt, es zum Beispiel eine »Tatsache «nennt, »daß die Wirtschaft die Arbeitskraft aller nicht mehr benötigt und immer weniger benötigen wird.« (S. 105)

3 Gorz vergißt immer wieder, daß kapitalistische Unternehmen ihre Profite nicht aus der Nicht-Verwendung, sondern aus der Ausbeutung von Arbeitskraft gewinnen. Zum Beispiel: »Schließlich liegt der Zweck von fast foods, Haushaltsrobotern und -computern, Expressreinigungen und -frisiersalons nicht darin, Arbeit zu schaffen, sondern sie einzusparen.« (S. 17) Man sollte fragen, wer hierbei welche Zwecke verfolgt.

4 Daraus allein könnte natürlich aus schon genannten Gründen eine langfristig abnehmende Nachfrage nach Arbeit nicht abgeleitet werden, sondern es wären zusätzlich Annahmen über Sättigungsgrenzen des Konsums zu treffen.

5 Gorz sagt zum Beispiel:»Die Arbeit und die Arbeitsgesellschaft sind nicht deshalb in Krise, weil es nicht genügend zu tun gäbe, sondern weil die Arbeit in einem sehr spezifischen Sinne knapp geworden ist und das, was zu tun ist, nur zu einem immer geringeren Teil noch zu dieser Arbeit gehört. Die Krise der Arbeit und der Arbeitsgesellschaft wird weder durch die Zunahme der Arbeit von Schuhputzern überwunden werden können, wie dies George Gilder glaubte; noch durch die wachsende Anzahl von Dienstboten, Hostessen und Windschutzscheiben-Putzern, wie dies Philippe Seguin behauptete; noch durch die Zunahme der Anzahl von Hausfrauen/männern, Prostituierten, Tagesmüttern oder -vätern, Reiseführern und Disneylands. Nicht weil all diese Leute nicht `arbeiteten<; was sie tun, hat nicht denselben Sinn wie die Arbeit im ökonomischen Simn und es ist nicht ungefährlich, ihre Tätigkeit jener gleichzustellen.« (S. 217 f.)

6 Viele der von Gorz vorgetragenen Überlegungen könnten wohl am besten als Versuche verstanden werden, ethische Kriterien für ökonomische Rationalisierungsprozesse zu formulieren; durchaus auch in der Tradition der historischen Schule der Nationalökonomie, zum Beispiel Roschers (Grundlagen der Nationalökonomie (23. Auflage), Stuttgart 1897, S. 2): »Giter nennen wir alles dasjenige, was zur mittelbaren oder unmittelbaren Befriedigung eines wahren menschlichen Bedürfnisses anerkannt brauchbar ist. « Roscher fügte hinzu; »Der Zusat $z$ > wahr < scheidet nicht allein dasjenige, was nur unvernünftige und unsittliche Bedürfnisse befriedigen könnte, vom Reiche der Güter aus, sondern vindiziert auch gleich den Grundbegriff der ganzen Volkswirtschaftslehre als einen Gegenstand ebenso wohl ethischer, wie psychologischer Untersuchung.《

7 Gorz' Ausführungen erinnern gelegentlich an die in der marxistischen Theorietradition häufiger vertretene Meinung, daß in einer kapitalistischen Gesellschaft eine Teilnahme am »gesellschaftlichen Leben« primär durch eine Mitwirkung an der gesellschaftlichen Warenproduktion, insbesondere durch Erwerbstätigkeit stattfindet. Diese offensichtlich empirisch falsche Auffassung wird von ihm schließlich normativ gewendet, um damit zu begründen, daß Einkommensansprüche durch Arbeitsverpflichtungen gesichert werden sollten: »Denn (...) man gehört als Mitglied zur Gesellschaft (genauer gesagt: zur modemen demokratischen und nicht zu einer Sklavenhaltergesellschaft), man hat ihr gegenüber Rechte, oder ist umgekehrt aus ihr teilweise ausgeschlossen, je nachdem, ob man am gesamtgesellschaftlich organisierten Produktionsprozeß teilnimmt oder nicht.« (S. 292) M.E. wäre es erforderlich, diesen Gedanken, demokratische Rechte von Arbeit und Vermögen abhängig zu machen, einer grundsätzlichen Kritik zu unterziehen.

8 An anderer Stelle (Zur politischen Ökonomie der Hausarbeit, Leviathan 1985/2, S. 187 ff.) habe ich mich bemüht, die Bedeutung dieser Unterscheidung im Hinblick auf die Frage ökonomischer Rationalisierbarkeit der Hausarbeit etwas genauer sichtbar zu machen. 
9 An diesem Beispiel zeigt sich zugleich, warum Formulierungen, die mit (Steigerungsmöglichkeiten) »physischer Produktivität« argumentieren, so leicht irreführend sind. Um diesen Begriff verwenden zu können, ist man gezwungen, den »Charakter « der Tätigkeiten bzw. ihrer Produkte zu definieren; dabei wird jedoch leicht übersehen, daß eine besonders wichtige Methode der Produktivitätssteigerung darin liegt, den »Charakter« der Tätigkeiten bzw. ihrer Produkte zu verändern. Es ist deshalb häufig angemessener, davon zu sprechen, daß der »Charakter« der Arbeitsprozesse bzw. der durch sie produzierten Gebrauchswerte aus weitgehend beliebig konzipierbaren Methoden zur Produktivitätssteigerung resultiert und insoweit nicht Voraussetzung, sondern ein Ergebnis ökonomischer Rationalisierung ist. 